\title{
A Robust FLOM Based Spectrum Sensing Scheme under Middleton Class A Noise in IoT
}

\author{
Enwei Xu, ${ }^{1}$ Shuo Shi, ${ }^{1}$ Dezhi Li, ${ }^{1}$ Xuemai Gu, ${ }^{1}$ and Fabrice Labeau ${ }^{2}$ \\ ${ }^{1}$ School of Electronics and Information Engineering, Harbin Institute of Technology, Harbin 150001, China \\ ${ }^{2}$ Department of Electrical and Computer Engineering, McGill University, Montreal, QC, Canada H3A 0G4 \\ Correspondence should be addressed to Xuemai Gu; guxuemai@hit.edu.cn
}

Received 14 December 2016; Accepted 16 March 2017; Published 6 April 2017

Academic Editor: Tao Han

Copyright (C) 2017 Enwei Xu et al. This is an open access article distributed under the Creative Commons Attribution License, which permits unrestricted use, distribution, and reproduction in any medium, provided the original work is properly cited.

Accessibility to remote users in dynamic environment, high spectrum utilization, and no spectrum purchase make Cognitive Radio (CR) a feasible solution of wireless communications in the Internet of Things (IoT). Reliable spectrum sensing becomes the prerequisite for the establishment of communication between IoT-capable objects. Considering the application environment, spectrum sensing not only has to cope with man-made impulsive noises but also needs to overcome noise fluctuations. In this paper, we study the Fractional Lower Order Moments (FLOM) based spectrum sensing method under Middleton Class A noise and incorporate a Noise Power Estimation (NPE) module into the sensing system to deal with the issue of noise uncertainty. Moreover, the NPE process does not need noise-only samples. The analytical expressions of the probabilities of detection and the probability of false alarm are derived. The impact on sensing performance of the parameters of the NPE module is also analyzed. The theoretical analysis and simulation results show that our proposed sensing method achieves a satisfactory performance at low SNR.

\section{Introduction}

The Internet of Things (IoT) has to construct comprehensive connections among variety of objects distributed over an extensive area. So the resources allocation to this large number of objects has to be resolved carefully to maintain a satisfactory Quality-of-Service (QoS) [1]. Generally, the frequency spectrum is one of the most important resources in wireless communications, and the problem of spectrum scarcity is getting worse as a result of the large number of applications [2]. Therefore, the available spectrum has to be carefully utilized by the IoT to ensure plenty of reliable connections between different objects. Fortunately, Cognitive Radio (CR) allows unlicensed users to utilize licensed bands opportunistically and enable them to reuse the frequency bands that are not heavily occupied by Primary Users (PU). Being able to address the spectrum scarcity issue, $\mathrm{CR}$ as a promising solution to exploit the available spectrum for the IoT has been proposed [3-5]. The ability of spectrum sensing to measure or sense the presence and absence of PU signal is essential because the operation of CR starts with detecting spectrum holes [6]. Spectrum Sensing methods proposed for identifying the presence of signal transmissions can be categorized as energy detector (ED) based sensing [7], matched filtering based sensing [8], waveform based sensing [9], cyclostationarity based sensing [10], radio identification based sensing [11], and so forth. Among these methods, ED based approaches are the most commonly used because of their low computational and implementation complexities [12].

Most of the previous studies on spectrum sensing only focused on signals contaminated by Additive White Gaussian Noise (AWGN). However, this assumption fails to model the behavior of certain noise types in IoT applications. Considering the applications of IoT such as Machine to Machine (M2M) networks and smart grids, a key challenge in establishing the IoT is wireless communication in the vicinity of vehicles, machines, or electrical power equipment which often radiates electromagnetic waves from switching power electronics components. In particular, this kind of waves in the form of impulse noise and high power transients disrupt wireless communication [3, 13]. Middleton Class A noise model is one of the widely investigated statistical distributions that are used to model this kind of man-made interference and the narrow band impulsive noise in different 
systems [14]. Being different from AWGN hypothesis, ED based detector is no longer an optimal detector and it has poor performance. Besides, Generalized Likelihood Ratio Test (GLRT) based detector as the optimal detector has a very complex structure, which will be explained later, when it is used under Middleton Class A noise. Recently, a large number of spectrum sensing approaches under different non-Gaussian noises have been proposed [15-17]. However, the implementation of these detectors in the IoT remains challenging because multiple antennas were used or the noise uncertainty was not considered.

Fractional Lower Order Moments (FLOM) demonstrated its capability in signal processing under non-Gaussian noise in [18]. When FLOM is applied to spectrum sensing, the test statistic has a similar expression as that of ED based sensing. Nevertheless, determination of the threshold also depends on the noise parameters in FLOM based sensing as in the case of ED based sensing, and a small noise uncertainty will cause significant performance loss $[19,20]$. To this end, a robust FLOM based sensing method should be studied as a promising solution of spectrum sensing under Middleton Class A noise, especially at low SNR. As is shown in our previous work [21], uncertainty of noise power is really destructive, while small estimation errors on other parameters of the noise do not have a strong effect on spectrum sensing performance. So in this paper, the focus is put on the spectrum sensing method under Middleton Class A noise with Noise Power Estimation (NPE), making the following contributions.

(i) We study the problem of spectrum sensing under Middleton Class A noise adopting FLOM based detector and derive the analytical expressions of the probability of false alarm $P_{\mathrm{fa}}$ and the probability of detection $P_{d}$. Then, we analyze the relationship between the sensing performance enhancement and the noise parameters.

(ii) We propose such an NPE based structure to deal with the issue of noise uncertainty that noise-only samples are not necessary in the estimation process. The performance of the proposed structure is analyzed, which relates the accuracy of the estimator to the estimation duration and the order of the estimator.

The following parts of this paper are organized as follows. The signal and noise models are defined in Section 2. In Section 3, FLOM are introduced to spectrum sensing as a suboptimal detector. The NPE based sensing structure is proposed in Section 4 and the derivation and analysis are presented in the same section. Section 5 includes the simulation and the results, and the conclusion is drawn in Section 6.

\section{Signal and Noise Models}

In spectrum sensing, the PU signal to be sensed is considered as a random process (called Bayesian model) in some works; and it is also considered as an unknown deterministic signal (called classical model) in others [22]. Lacking in the knowledge of the PU signals, we choose Bayesian model and consider a source $x$ with a zero-mean Gaussian probability density function (pdf)

$$
f_{X}(x)=G\left(x ; \sigma_{X}^{2}\right)=\frac{1}{\sqrt{2 \pi} \sigma_{X}} e^{-x^{2} / 2 \sigma_{X}^{2}}
$$

and $x$ is transmitted over a channel impaired by a Middleton Class A noise $z$, whose pdf is

$$
f_{Z}(z)=\sum_{m=0}^{\infty} \beta_{m} G\left(z ; \sigma_{m}^{2}\right)=\sum_{m=0}^{\infty} \frac{\beta_{m}}{\sqrt{2 \pi} \sigma_{m}} e^{-z^{2} / 2 \sigma_{m}^{2}},
$$

where $\beta_{m}=e^{-A} A^{m} / m$ ! indicates that $m$ noise sources contribute to the impulsive event simultaneously and $A=$ $\mathscr{E}\{m\}=\sum_{m=0}^{\infty} m \beta_{m}$ is the corresponding overlap index denoting the average number of impulse noise sources active at any given time. Larger values of $A$ make the characteristic of the noise closer to Gaussian noise. Moreover, $\sigma_{Z}^{2}=\mathscr{E}\left\{z^{2}\right\}=\sigma_{G}^{2}+$ $\sigma_{I}^{2}$ is the noise power, in which $\sigma_{G}^{2}$ is the Gaussian power and $\sigma_{I}^{2}$ is the impulsive power. $T=\sigma_{G}^{2} / \sigma_{I}^{2}$ is the power ratio of the Gaussian component to the impulsive component, and $\sigma_{m}^{2}=$ $((m / A+T) /(1+T)) \sigma_{Z}^{2}=\sigma_{G}^{2}+\sigma_{I}^{2}(m / A)$. Thus, the Middleton Class A noise is totally characterized by the parameters $A, T$, and $\sigma_{Z}^{2}$. In addition, the PU signal and the noise are assumed to be mutually independent and SNR is defined by $\sigma_{X}^{2} / \sigma_{Z}^{2}$.

\section{Spectrum Sensing under Middleton Class A Noise}

Depending on the idle state and busy state of the PU, with the presence of the noise, detecting the presence of PU is usually considered as the following binary hypothesis testing problem [23]:

$$
\begin{aligned}
& H_{0}: y(n)=z(n) \quad \text { PU is absent, } \\
& H_{1}: y(n)=x(n)+z(n) \quad \text { PU is present }
\end{aligned}
$$

in which $n=1,2,3, \ldots, N, N$ is the number of observed samples; $y(n)$ is the signal observed by sensing receiver with $x(n)$ and $z(n)$ denoting the PU signal and the additive impulsive noise respectively. $H_{0}$ means that the PU signal is absent and $H_{1}$ means that the PU signal is present.

According to the Neyman-Pearson (NP) theorem, GLRT can maximize detection probability when the probability of false alarm is fixed. So we attempted to use GLRT as an optimal method first. With the signal and noise models described in Section 2, the globally optimal detector can be expressed as

$$
\Lambda_{\mathrm{GO}}=\log \frac{f_{Y \mid H_{1}}\left(\mathbf{y} \mid H_{1}\right)}{f_{Y \mid H_{0}}\left(\mathbf{y} \mid H_{0}\right)}=\sum_{n=1}^{N} \log \frac{f_{Y \mid H_{1}}\left(y(n) \mid H_{1}\right)}{f_{Y \mid H_{0}}\left(y(n) \mid H_{0}\right)},
$$

where $\mathbf{y}$ is the vector of the received samples. If $\Lambda_{\mathrm{GO}} \geq 0$, it means that the PU signal is present. Otherwise, it means that the PU signal is absent.

Substituting (3) into (4), we have

$$
\Lambda_{\mathrm{GO}}=\sum_{n=1}^{N} \log \frac{\mathscr{E}_{X}\left\{f_{Z}(y(n)-x(n))\right\}}{f_{Z}(y(n))},
$$

in which $\mathscr{E}\{\cdot\}$ denotes statistical expectation. 
Obviously, $\Lambda_{\mathrm{GO}}$ is with respect to the pdf of the PU signal which may not be obtained by unlicensed users. However, CR always operates in low SNR (i.e., $\sigma_{X}^{2} / \sigma_{Z}^{2} \rightarrow 0$ ) circumstance, especially in IoT. By making the low SNR assumption, we can obtain a locally optimal detector from the globally optimal detector.

Equation (5) can be simplified by using Taylor series [24],

$$
f_{Z}(y-x) \approx f_{Z}(y)-x f_{Z}^{\prime}(y)+\frac{1}{2} x^{2} f_{Z}^{\prime \prime}(y),
$$

where we drop the time dependence $n$ for clarity.

The second term of the right side in (6) equals 0 when taking the expectation. Hence,

$$
\mathscr{E}_{X}\left\{f_{Z}(y(n)-x(n))\right\}=f_{Z}(y(n))+\frac{\sigma_{X}^{2}}{2} f_{Z}^{\prime \prime}(y(n)),
$$

where we assume $\mathscr{E}\left\{x^{2}\right\}=\sigma_{X}^{2}$.

Then the locally optimal detector under low SNR can be expressed as

$$
\begin{aligned}
\Lambda_{\mathrm{LO}} & =\sum_{n=1}^{N} \log \left(1+\frac{\sigma_{X}^{2}}{2} \frac{f_{Z}^{\prime \prime}(y(n))}{f_{Z}(y(n))}\right) \\
& \approx \frac{\sigma_{X}^{2}}{2} \sum_{n=1}^{N} \frac{f_{Z}^{\prime \prime}(y(n))}{f_{Z}(y(n))} .
\end{aligned}
$$

Here we use $\log (1+x) \approx x$ for $x \rightarrow 0$. Obviously, this locally optimal detector only requires the pdf of the noise.

For the case of AWGN, the differential part of (8) can be simplified into

$$
g(y(n))=\frac{f_{Z}^{\prime \prime}(y(n))}{f_{Z}(y(n))}=\frac{|y(n)|^{2}}{\sigma_{Z}^{4}}-\frac{1}{\sigma_{Z}^{2}} \propto|y(n)|^{2} .
$$

The locally optimal detector equals the traditional ED. Unfortunately, $g(y(n))$ cannot be easily simplified under Middleton Class A noise hypothesis. From (8), it can be seen that the locally optimal GLRT detector is with respect to the pdf of the noise which contains infinite summation. Moreover, the structure of the detector has to change with the change of the noise parameters which makes the implementation of GLRT detector impossible. Consequently, $g(y(n))$ should be converted into a simpler nonlinear operator so that it can be implemented practically.

Under impulsive noise hypothesis, the presence of impulses increases the false alarm during sensing process. To improve the sensing performance, the impact of randomly appearing large amplitudes in the noise should be reduced. Inspired by the capability of FLOM in signal processing under non-Gaussian noise and the expression of energy detector, we use FLOM as a suboptimal detector and the corresponding test statistic is given in

$$
\Lambda_{\mathrm{SO}}(y)=\frac{1}{N} \sum_{n=1}^{N}|y(n)|^{p}
$$

where $0<p<2$.

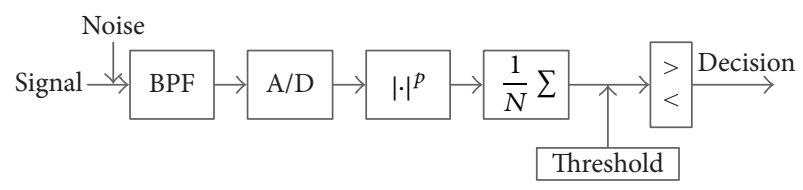

FIgURE 1: Structure of FLOM based detector.

Through fractional power operation, a nonlinear operation, the large impulse amplitude in the noise can be reduced, while the small values almost remain unchanged. As a result, a good sensing performance can be obtained. In addition, with the similar expression of ED, FLOM based detector can be implemented practically when the parameter $p$ is determined.

\section{FLOM Based Spectrum Sensing with Noise Power Estimation}

4.1. FLOM Based Spectrum Sensing. As for the FLOM based detector, the structure is shown in Figure 1. According to the central limit theorem [25], when $N$ is large enough, the metric $\Lambda_{\mathrm{SO}}(y)$ in (10) can be approximated as a Gaussian random variable,

$$
\begin{gathered}
\left.\Lambda_{\mathrm{SO}}(y)\right|_{H_{0}} \sim \mathcal{N}\left(\mu_{0, p}, \frac{\sigma_{0, p}^{2}}{N}\right), \\
\left.\Lambda_{\mathrm{SO}}(y)\right|_{H_{1}} \sim \mathcal{N}\left(\mu_{1, p}, \frac{\sigma_{1, p}^{2}}{N}\right)
\end{gathered}
$$

in which

$$
\begin{aligned}
\mu_{0, p} & =\mathscr{E}\left\{|y(n)|^{p} \mid H_{0}\right\}, \\
\mu_{1, p} & =\mathscr{E}\left\{|y(n)|^{p} \mid H_{1}\right\}, \\
\sigma_{0, p}^{2} & =\mathscr{E}\left\{\left(|y(n)|^{p}-\mu_{0, p}\right)^{2} \mid H_{0}\right\} \\
& =\mathscr{E}\left\{|y(n)|^{2 p} \mid H_{0}\right\}-\mu_{0, p}^{2}, \\
\sigma_{1, p}^{2} & =\mathscr{E}\left\{\left(|y(n)|^{p}-\mu_{1, p}\right)^{2} \mid H_{1}\right\} \\
& =\mathscr{E}\left\{|y(n)|^{2 p} \mid H_{1}\right\}-\mu_{1, p}^{2} .
\end{aligned}
$$

The pth order central absolute moments of a Gaussian random variable $S \sim \mathcal{N}\left(0, \sigma^{2}\right)$ can be expressed as

$$
\mathscr{E}\left[|S|^{p}\right]=\frac{2^{p / 2}}{\sqrt{\pi}} \Gamma\left(\frac{p+1}{2}\right) \sigma^{p},
$$

where $\Gamma(\cdot)$ is the usual gamma function defined by $\Gamma(x)=$ $\int_{0}^{\infty} t^{x-1} e^{-t} d t$. 
Assuming that the noise $z(n)$ and the PU signal $x(n)$ are independent of each other, we can obtain that

$$
\begin{aligned}
\left.f_{Y}(y(n))\right|_{H_{0}} & =f_{Z}(y(n))=\sum_{m=0}^{\infty} \frac{\beta_{m}}{\sqrt{2 \pi} \sigma_{m}} e^{-y(n)^{2} / 2 \sigma_{m}^{2}}, \\
\left.f_{Y}(y(n))\right|_{H_{1}} & =f_{X}(y(n)) * f_{Z}(y(n)) \\
& =\sum_{m=0}^{\infty} \frac{\beta_{m}}{\sqrt{2 \pi\left(\sigma_{m}^{2}+\sigma_{X}^{2}\right)}} e^{-y(n)^{2} / 2\left(\sigma_{m}^{2}+\sigma_{X}^{2}\right)} .
\end{aligned}
$$

Then it can be easily concluded that

$$
\begin{aligned}
& \mu_{0, p}=\frac{2^{p / 2}}{\sqrt{\pi}} \Gamma\left(\frac{p+1}{2}\right) \sum_{m=0}^{\infty} \beta_{m} \sigma_{m}^{p}, \\
& \mu_{1, p}=\frac{2^{p / 2}}{\sqrt{\pi}} \Gamma\left(\frac{p+1}{2}\right) \sum_{m=0}^{\infty} \beta_{m}{\sqrt{\sigma_{m}^{2}+\sigma_{X}^{2}}}^{p}, \\
& \sigma_{0, p}^{2}=\frac{2^{p}}{\sqrt{\pi}} \Gamma\left(\frac{2 p+1}{2}\right) \sum_{m=0}^{\infty} \beta_{m} \sigma_{m}^{2 p}-\mu_{0, p}^{2}, \\
& \sigma_{1, p}^{2}=\frac{2^{p}}{\sqrt{\pi}} \Gamma\left(\frac{2 p+1}{2}\right) \sum_{m=0}^{\infty} \beta_{m}\left(\sigma_{m}^{2}+\sigma_{X}^{2}\right)^{p}-\mu_{1, p}^{2} .
\end{aligned}
$$

Figure 2 demonstrates the distribution of $\left.\Lambda(y)\right|_{H_{0}}$ and $\left.\Lambda(y)\right|_{H_{1}}$ in ED based sensing and FLOM based sensing at SNR $=-5 \mathrm{~dB}$, respectively. Here we choose $A=T=0.1$, $p=0.4$, and $N=100$ as an example. The two distribution curves in FLOM are more clearly separated than those of ED. It intuitively explained why the performance of FLOM based sensing is much better than ED based sensing under Middleton Class A noise.

The optimal value of $p$ can be determined by the deflection coefficient which is usually defined as (16) to measure the performance for binary hypothesis testing problems [26].

$$
d(p)=\frac{\mu_{1, p}-\mu_{0, p}}{\sigma_{0, p}} .
$$

The probability of false alarm $P_{\mathrm{fa}}$ and probability of detection $P_{d}$ can be given in terms of the $Q$ function by

$$
\begin{gathered}
P_{\mathrm{fa}}=P_{r}\left(\Lambda_{\mathrm{SO}}(y)>\gamma \mid H_{0}\right)=Q\left(\frac{\gamma-\mu_{0, p}}{\sqrt{\sigma_{0, p}^{2} / N}}\right), \\
P_{d}=P_{r}\left(\Lambda_{\mathrm{SO}}(y)>\gamma \mid H_{1}\right)=Q\left(\frac{\gamma-\mu_{1, p}}{\sqrt{\sigma_{1, p}^{2} / N}}\right),
\end{gathered}
$$

where $Q(\cdot)$ is defined by $Q(x)=(1 / \sqrt{2 \pi}) \int_{x}^{\infty} \exp \left(-t^{2} / 2\right) d t$ and $\gamma$ is the threshold chosen for judging the presence or absence of the PU signal.

The threshold is normally chosen to satisfy a target $P_{\mathrm{fa} \_\mathrm{DES}}$ according to the Neyman-Pearson criterion in practice [27], which requires the noise parameters to be known, as shown in

$$
\gamma=\sqrt{\frac{\sigma_{0, p}^{2}}{N}} Q^{-1}\left(P_{\text {fa_DES }}\right)+\mu_{0, p} .
$$

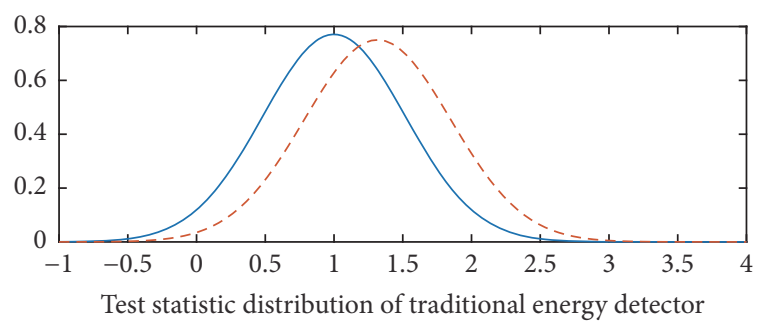

$-H 0$

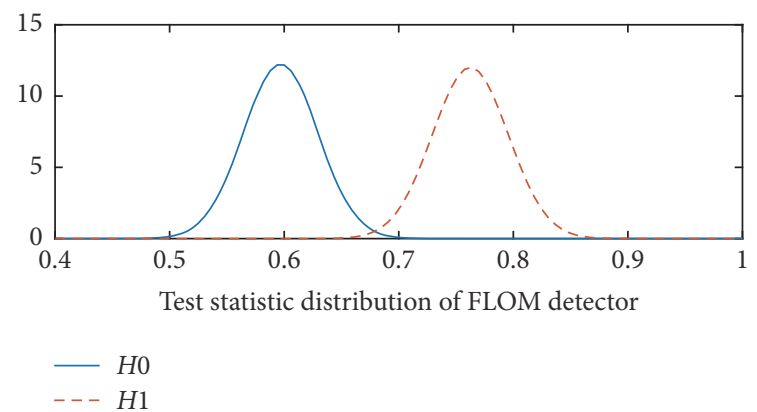

FIGURE 2: Distribution comparison of the test statistics of FLOM detector and energy detector with $A=T=0.1, p=0.4$, and $N=100$, at $\mathrm{SNR}=-5 \mathrm{~dB}$.

Substituting (18) into the second equation of (17), we can obtain the final probability of detection.

$$
P_{d}=Q\left(\frac{\sqrt{\sigma_{0, p}^{2} / N} Q^{-1}\left(P_{\mathrm{fa}_{-} \mathrm{DES}}\right)+\mu_{0, p}-\mu_{1, p}}{\sqrt{\sigma_{1, p}^{2} / N}}\right) .
$$

As shown in (19), increasing the number of the samples $N$ can improve the sensing ability but will take longer time. So in practice, compromise should be made between sensing performance and processing time.

4.2. FLOM Detector with Noise Power Estimation. As mentioned above, FLOM based sensing outperforms ED based methods. However, the noise power fluctuation is generally encountered in practice. So the noise power uncertainty should be considered to ensure the implementation of the sensing method. If the estimated noise power is assumed to be in an interval $\widehat{\sigma}_{Z}^{2} \in\left[(1 / \rho) \sigma_{Z}^{2}, \rho \sigma_{Z}^{2}\right], \rho>1$ is a parameter that quantifies the amount of the uncertainty. To achieve a target $P_{\mathrm{fa}}$ and $P_{d}$ robustly, the following equations need to hold:

$$
\begin{gathered}
P_{\mathrm{fa}}=\max _{\hat{\sigma}_{Z}^{2} \in\left[(1 / \rho) \sigma_{Z}^{2}, \rho \sigma_{Z}^{2}\right]} Q\left(\frac{\gamma-\mu_{0, p}}{\sqrt{\sigma_{0, p}^{2} / N}}\right), \\
P_{d}=\min _{\hat{\sigma}_{Z}^{2} \in\left[(1 / \rho) \sigma_{Z}^{2}, \rho \sigma_{Z}^{2}\right]} Q\left(\frac{\gamma-\mu_{1, p}}{\sqrt{\sigma_{1, p}^{2} / N}}\right) .
\end{gathered}
$$




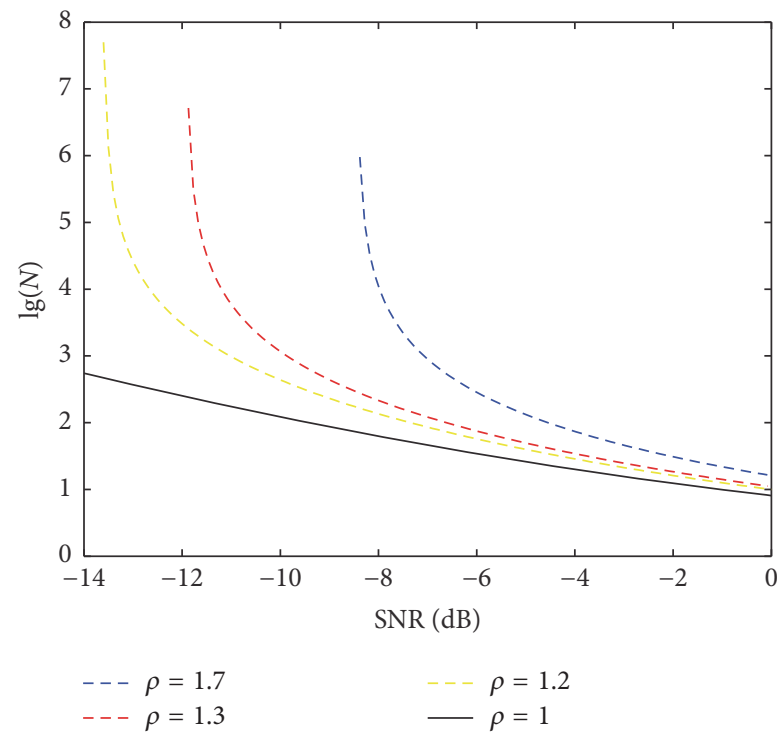

Figure 3: SNR walls with different $\rho$.

Due to the $Q$ function being a monotonically decreasing function, we obtain

$$
\begin{aligned}
& P_{\mathrm{fa}}=\left.Q\left(\frac{\gamma-\mu_{0, p}}{\sqrt{\sigma_{0, p}^{2} / N}}\right)\right|_{\widehat{\sigma}_{Z}^{2}=\rho \sigma_{Z}^{2}}, \\
& P_{d}=\left.Q\left(\frac{\gamma-\mu_{1, p}}{\sqrt{\sigma_{1, p}^{2} / N}}\right)\right|_{\widehat{\sigma}_{Z}^{2}=(1 / \rho) \sigma_{Z}^{2}} .
\end{aligned}
$$

From (21), we have

$$
N=\left[\frac{Q^{-1}\left(P_{\mathrm{fa}}\right) \sigma_{0, p}-Q^{-1}\left(P_{d}\right) \sigma_{1, p}}{\mu_{1, p}-\mu_{0, p}}\right]^{2} .
$$

Figure 3 displays how many samples are needed to achieve target $P_{\mathrm{fa}}=0.1$ and $P_{d}=0.9$ under different SNR with $A=T=0.1$ (note that the vertical axis is a logarithmic scale). It is obvious that the number of samples which needed $N$ will tend to $\infty$ when SNR approaches a specific value with any $\rho>1$, which is called SNR Wall phenomenon. And the SNR wall will become higher with the uncertainty $\rho$ becoming higher. It means that the detector cannot robustly detect the signal if the signal-noise power ratio is lower than the specific value. So the performance of an FLOM detector with a fixed threshold also degrades like ED when the noise power uncertainty occurs [19].

To keep a satisfactory sensing performance, the real time value of noise power should be used to determine the threshold in every sensing period. Fortunately, we found that the higher order moment of the samples can be a maximum likelihood (ML) noise power estimator, although it is not able to act as a good sensing statistic. In this paper, an adaptive threshold sensing structure is adopted with a higher order

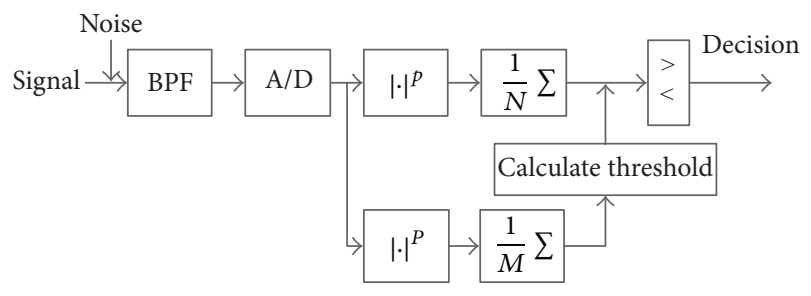

FIGURE 4: Structure of FLOM detector with noise power estimation module.

moment detector as a real time noise power estimator and updating thresholds during each sensing process. From the structure of the NPE based detector shown in Figure 4, it can be seen that the estimator uses the same samples as the sensor and noise-only samples are not necessary in the noise estimation process. Without the noise-only slots, the transmitter can make full use of time slots and further improves the spectrum utilization.

The statistic of the noise power estimator is shown in

$$
\mathrm{PW}=\frac{1}{M} \sum_{n=1}^{M}|y(n)|^{P}
$$

in which $P \geq 2$ is the higher order and $M \geq N$ is the estimation time window.

Due to the fact that the expectations of $|z(n)|^{P}$ and $\mid z(n)+$ $\left.x(n)\right|^{P}$ have very small differences at low SNR, this $P$ th order estimator can operate an unbiased estimation of the $P$ th order absolute moment of $z(n)$ no matter whether the PU signal is present or absent. The $P$ th order absolute moment of $z(n)$ is estimated by PW; that is,

$$
\frac{2^{P / 2}}{\sqrt{\pi}} \Gamma\left(\frac{P+1}{2}\right) \sum_{m=0}^{\infty} \beta_{m}\left(\frac{m / A+T}{1+T}\right)^{P / 2} \widehat{\sigma}_{Z}^{P}=\mathrm{PW}
$$

where $\sigma_{m}^{2}=((m / A+T) /(1+T)) \sigma_{Z}^{2}$ is substituted.

It is worth mentioning that the estimated noise power $\widehat{\sigma}_{Z}^{2}$ in the proposed estimator is not obtained directly when $P>$ 2 . But the value we need for calculating the threshold is $\widehat{\sigma}_{Z}$, so estimating $\widehat{\sigma}_{Z}^{2}$ or $\widehat{\sigma}_{Z}^{P}$ can both achieve this purpose. The only difference between them is the estimation accuracy which will be discussed in the following section.

Substituting (15) into (18) and replacing $\sigma_{Z}^{P}$ by the estimated $\widehat{\sigma}_{Z}^{P}$ in (24), the corresponding threshold can be obtained

$$
\begin{aligned}
\widehat{\gamma}=\left(\sqrt{\frac{\beta-\alpha^{2}}{N}} Q^{-1}\left(P_{\mathrm{fa} \_\mathrm{DES}}\right)+\alpha\right) \widehat{\sigma}_{Z}^{p}, & \\
\widehat{\sigma}_{Z}^{p} & =\left(\widehat{\sigma}_{Z}^{P}\right)^{p / P}=\left(\frac{\mathrm{PW}}{\delta}\right)^{p / P},
\end{aligned}
$$


where

$$
\begin{aligned}
& \alpha=\frac{2^{p / 2}}{\sqrt{\pi}} \Gamma\left(\frac{p+1}{2}\right) \sum_{m=0}^{\infty} \beta_{m}\left(\frac{m / A+T}{1+T}\right)^{p / 2}, \\
& \beta=\frac{2^{p}}{\sqrt{\pi}} \Gamma\left(\frac{2 p+1}{2}\right) \sum_{m=0}^{\infty} \beta_{m}\left(\frac{m / A+T}{1+T}\right)^{p}, \\
& \delta=\frac{2^{P / 2}}{\sqrt{\pi}} \Gamma\left(\frac{P+1}{2}\right) \sum_{m=0}^{\infty} \beta_{m}\left(\frac{m / A+T}{1+T}\right)^{P / 2} .
\end{aligned}
$$

Utilizing the threshold $\widehat{\gamma}$, the real probability of false alarm and probability of detection can be expressed as

$$
\begin{aligned}
& P_{\mathrm{fa} \_r} \\
& \quad=\int_{-\infty}^{\infty} f_{0}(\mathrm{pw}) \mathrm{Q}\left(\frac{\widehat{\gamma}\left(\mathrm{pw}, P_{\mathrm{fa} \_\mathrm{DES}}\right)-\mu_{0, p}}{\sqrt{\sigma_{0, p}^{2} / N}}\right) d \mathrm{pw}, \\
& P_{d \_r} \\
& \quad=\int_{-\infty}^{\infty} f_{1}(\mathrm{pw}) \mathrm{Q}\left(\frac{\widehat{\gamma}\left(\mathrm{pw}, P_{\mathrm{fa} \_ \text {DES }}\right)-\mu_{1, p}}{\sqrt{\sigma_{1, p}^{2} / N}}\right) d \mathrm{pw}
\end{aligned}
$$

in which $f_{0}(\cdot)$ and $f_{1}(\cdot)$ denote the pdf of PW under $H_{0}$ and $H_{1}$, respectively.

The previous discussion indicates that PW also obeys Gaussian distribution with a large $M$. So

$$
\begin{aligned}
& \mathrm{PW}_{H_{0}} \sim \mathcal{N}\left(\mu_{0, P}, \frac{\sigma_{0, P}^{2}}{M}\right), \\
&\left.\mathrm{PW}\right|_{H_{1}} \sim \mathcal{N}\left(\mu_{1, P}, \frac{\sigma_{1, P}^{2}}{M}\right),
\end{aligned}
$$

where

$$
\begin{aligned}
& \mu_{0, P}=\frac{2^{P / 2}}{\sqrt{\pi}} \Gamma\left(\frac{P+1}{2}\right) \sum_{m=0}^{\infty} \beta_{m} \sigma_{m}^{P}, \\
& \mu_{1, P}=\frac{2^{P / 2}}{\sqrt{\pi}} \Gamma\left(\frac{P+1}{2}\right) \sum_{m=0}^{\infty} \beta_{m}{\sqrt{\sigma_{m}^{2}+\sigma_{X}^{2}}}^{P}, \\
& \sigma_{0, P}^{2}=\frac{2^{P}}{\sqrt{\pi}} \Gamma\left(\frac{2 P+1}{2}\right) \sum_{m=0}^{\infty} \beta_{m} \sigma_{m}^{2 P}-\mu_{0, P}^{2}, \\
& \sigma_{1, P}^{2}=\frac{2^{P}}{\sqrt{\pi}} \Gamma\left(\frac{2 P+1}{2}\right) \sum_{m=0}^{\infty} \beta_{m}\left(\sigma_{m}^{2}+\sigma_{X}^{2}\right)^{P}-\mu_{1, P}^{2} .
\end{aligned}
$$

Substituting (28) and (29) into (27), the value of $P_{\mathrm{fa} \_r}$ and $P_{d \_r}$ can be got.

\section{Simulation and Results}

In this section, we give the simulations and analysis of the theoretical results in Section 4.

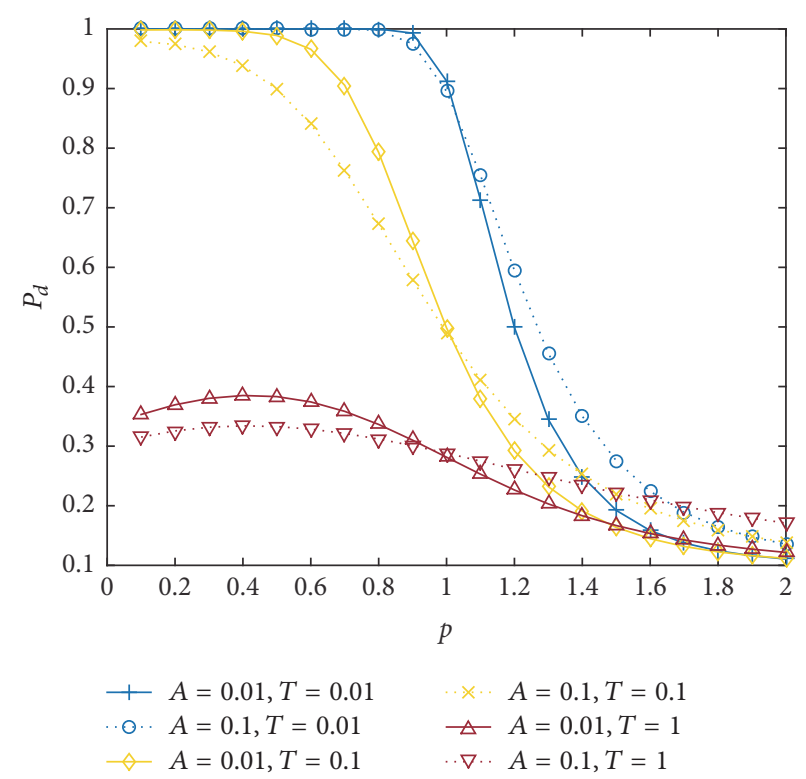

FIgure 5: Probability of detection $P_{d}$ versus $p$ for different $A$ and $T$ with target probability of false alarm $P_{\mathrm{fa} D E S}=0.1 ; N=1000$ at $\mathrm{SNR}=-15 \mathrm{~dB}$.

5.1. FLOM Based Spectrum Sensing. Let $P_{\mathrm{fa} \text { DES }}=0.1$ as the target probability of false alarm; Figure 5 shows the corresponding $P_{d}$ versus $p$ varying from 0.1 to 2 with $\mathrm{SNR}=$ $-15 \mathrm{~dB} ; N=1000$. It can be seen that traditional ED based sensing $(p=2)$ has very poor performance $\left(P_{d}<0.2\right)$ when SNR is as low as $-15 \mathrm{~dB}$. However, the FLOM detector shows large improvement and a satisfactory $P_{d}$ can be easily achieved when an appropriate value for $p$ is chosen, $p=0.4$ as an example. The result also indicates that the performance improvement also depends on $T$. The larger improvement will be obtained when $T$ is getting smaller. In other words, the FLOM detector is more suitable for the circumstances under which the noise is more impulsive.

From another perspective, Figure 6 shows several Receiver Operating Characteristic (ROC) curves of ED and FLOM based sensing in both theory and simulation with $A=$ $T=0.1 ; N=1000$ at SNR $=-15 \mathrm{~dB}$. The simulation results are well matched with theory results. Obviously, FLOM based sensing outperforms ED based one and a better performance will be achieved when the value of $p$ is smaller.

The detection ability of the FLOM detector under different SNR is plotted in Figure 7. The similar conclusion is drawn as those in Figures 5 and 6. In addition, take $P_{d}=0.9$ as a target probability of detection; FLOM based sensing can work with SNR as low as $-15 \mathrm{~dB}$ when $p=0.4$, but ED based sensing will only work when SNR is not lower than $-5 \mathrm{~dB}$.

5.2. FLOM Detector with Noise Power Estimation. The performance of the NPE based FLOM sensing approach must be related to the estimation accuracy, so the relationship between $P_{d_{-} r}, P_{\mathrm{fa} \_} r$, and the order $P$ of the estimator is given in Figure 8. Here we set a relatively low SNR that $\mathrm{SNR}=-20 \mathrm{~dB}$, in order to see the rate of change more clearly. The target probability of false alarm $P_{\text {fa_DES }}=0.1, N=1000, A=$ 


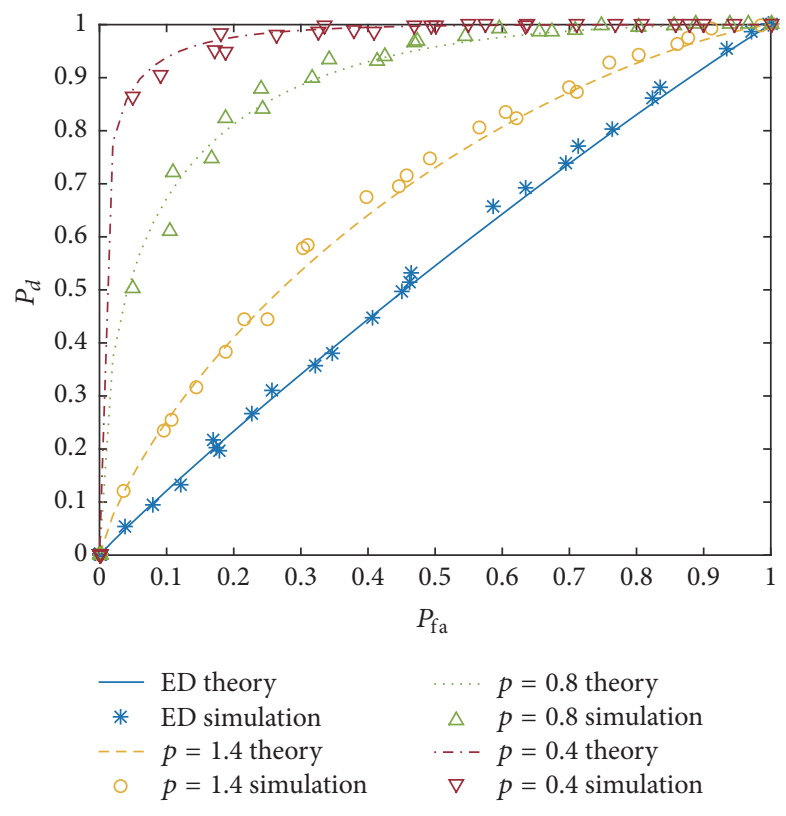

FIgURE 6: ROC curves of FLOM detector with $A=T=0.1 ; N=$ 1000 at $\mathrm{SNR}=-15 \mathrm{~dB}$.

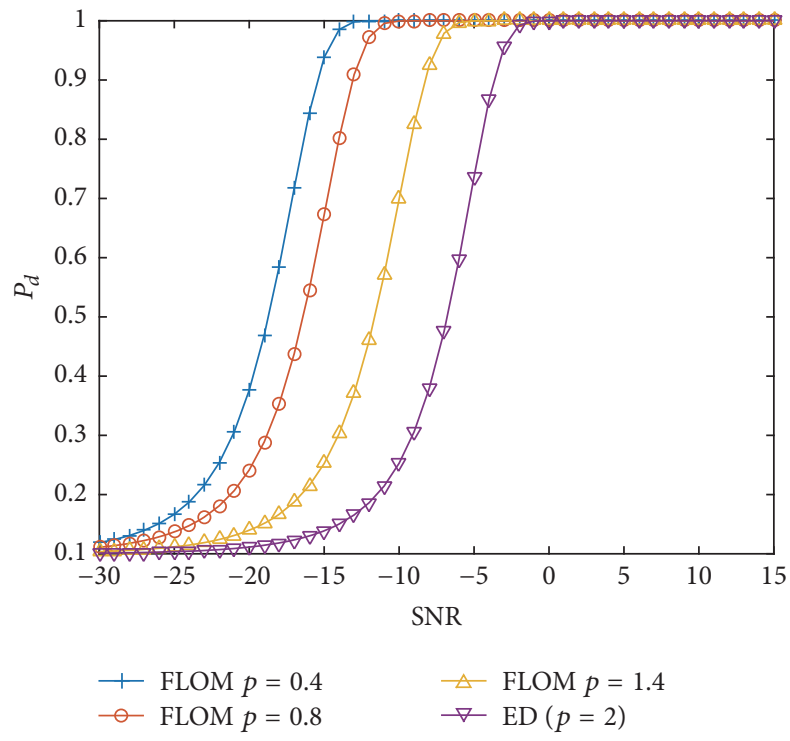

Figure 7: Probability of detection $P_{d}$ versus SNR for different $p$ with target probability of false alarm $P_{\mathrm{fa} \text { DES }}=0.1, A=T=0.1$, and $N=1000$.

$T=0.1$, and $p=0.4$. From Figure 8 , it can be seen that $P_{d_{-} r}$ and $P_{\mathrm{fa}_{-} r}$ are both increasing with different slopes as the value of $P$ increases, but the change of $P_{\mathrm{fa} r}$ is slight. After $P \geq 3, P_{d_{-} r}$ and $P_{\mathrm{fa}_{-} r}$ tend to be stable. Moreover, the order of the estimator can be set as $P=2$ as a tradeoff to reduce computational complexity.

Figure 9 demonstrates the theory and the simulation results of $P_{d_{-} r}$ and $P_{\mathrm{fa}_{-} r}$ versus SNR of our proposed FLOM detector. The threshold $\widehat{\gamma}$ is calculated using $\widehat{\sigma}_{Z}^{P}$ and the target probability of false alarm $P_{\mathrm{fa} \_ \text {DES }}=0.1, N=1000, A=$ $T=0.1, p=0.4$, and $P=2$. In these curves, ideal FLOM
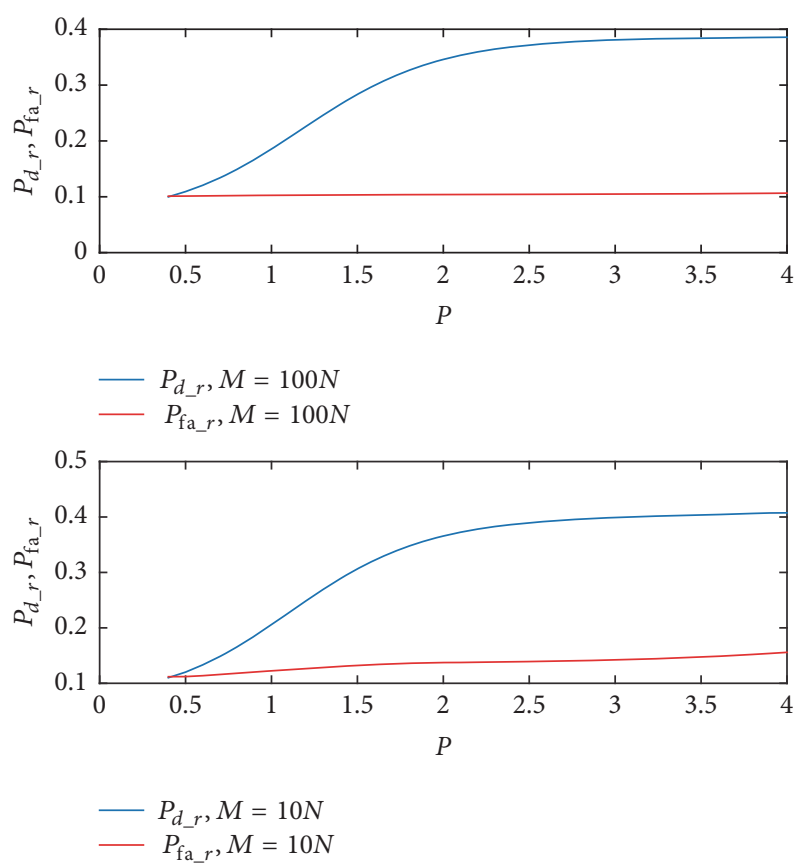

FIguRe 8: $P_{d_{r} r}$ and $P_{\mathrm{fa} r}$ versus the estimator order $P$ at $\mathrm{SNR}=$ $-20 \mathrm{~dB}$ with target probability of false alarm $P_{\mathrm{fa} \_ \text {DES }}=0.1, N=1000$, $A=T=0.1$, and $p=0.4$.

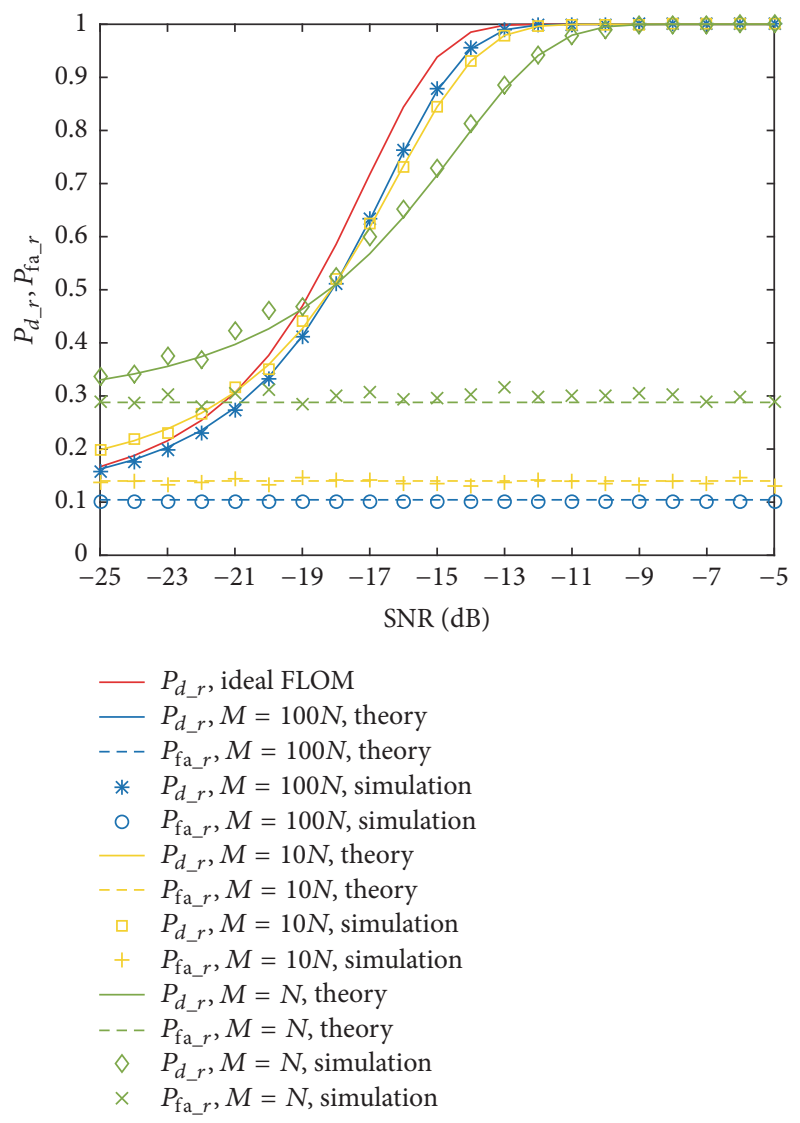

FIGURE 9: Ideal probability of detection $P_{d}$, theory, and simulation of $P_{d r}$ and $P_{\mathrm{fa}} r$ versus SNR with target probability of false alarm $P_{\mathrm{fa} \_ \text {DES }}=0.1, N=1000, A=T=0.1, p=0.4$, and $P=2$. 
represents the noise power perfectly known. It is obvious that a better sensing performance can be obtained when the estimation time window $M$ is getting larger, while it also takes a longer processing time and the performance improvement will slow down when $M$ is larger than $10 N$.

It is worth mentioning that the number of observed samples has impact on the energy consumption and the sensing time. As an example, the proposed sensing structure works well at SNR $=-15 \mathrm{~dB}$ with the set of parameters ( $A=T=0.1)$ using in the simulation. In practice, the noise power ratio $T$ usually varies within $\left[10^{-4}, 0.1\right]$. Figures 5 and 7 indicate that the better performance will be obtained when $T$ is getting smaller and SNR is getting larger. So in the case that SNR is not as low as $-15 \mathrm{~dB}$ or the noise is more impulsive ( $T \ll 0.1$ ), the number of the observed samples could be drastically reduced. As a result, the energy consumption and the sensing time will be reduced and the proposed detector can be applied in IoT devices.

\section{Conclusion}

Cognitive Radio can be a helpful technology for utilizing and allocating frequency spectrum in the IoT. The utilization of the FLOM can successfully achieve spectrum sensing task for CR under Middleton Class A noise in the IoT. We derive the analytical expressions of the probability of false alarm $P_{\mathrm{fa}}$ and the probability of detection $P_{d}$ demonstrating the relationship between the performance enhancement and the value of the fractional order $p$. For all circumstances, reasonable choices of $p$ can be made to achieve a satisfactory performance using the deflection coefficient. After an NPE module being added to the sensing system, the problem of noise uncertainty at low SNR has been resolved. Both the power estimation time window $M$ and the order $P$ of the NPE module impact sensing performance. By choosing reasonable $M$ and $P$, simulation shows that our proposed sensing scheme has almost the same performance as that when the noise power is perfectly known.

\section{Conflicts of Interest}

The authors declare that there are no conflicts of interest regarding the publication of this paper.

\section{Acknowledgments}

This work was supported by Hydro-Quebec, the Natural Sciences and Engineering Research Council of Canada, and McGill University in the framework of the NSERC/HydroQuebec/McGill Industrial Research Chair in Interactive Information Infrastructure for the Power Grid.

\section{References}

[1] A. Ali and W. Hamouda, "Advances on spectrum sensing for cognitive radio networks: theory and applications," IEEE Communications Surveys \& Tutorials, 2016.

[2] N. Zhang, H. Zhou, K. Zheng, N. Cheng, J. W. Mark, and X. S. Shen, "Cooperative heterogeneous framework for spectrum harvesting in cognitive cellular network," IEEE Communications Magazine, vol. 53, no. 5, pp. 60-67, 2015.

[3] A. Ali, W. Hamouda, and M. Uysal, "Next generation M2M cellular networks: challenges and practical considerations," IEEE Communications Magazine, vol. 53, no. 9, pp. 18-24, 2015.

[4] A. Aijaz and A. H. Aghvami, "Cognitive machine-to-machine communications for internet-of-things: a protocol stack perspective," IEEE Internet of Things Journal, vol. 2, no. 2, pp. 103112, 2015.

[5] R. Deng, J. Chen, X. Cao, Y. Zhang, S. Maharjan, and S. Gjessing, "Sensing-performance tradeoff in cognitive radio enabled smart grid," IEEE Transactions on Smart Grid, vol. 4, no. 1, pp. 302-310, 2013.

[6] T. Yücek and H. Arslan, "A survey of spectrum sensing algorithms for cognitive radio applications," IEEE Communications Surveys and Tutorials, vol. 11, no. 1, pp. 116-130, 2009.

[7] C. Liu, M. Li, and M.-L. Jin, "Blind energy-based detection for spatial spectrum sensing," IEEE Wireless Communications Letters, vol. 4, no. 1, pp. 98-101, 2015.

[8] X. Z. Zhang, F. F. Gao, R. Chai, and T. Jiang, "Matched filter based spectrum sensing when primary user has multiple power levels," China Communications, vol. 12, no. 2, pp. 21-31, 2015.

[9] M. Iqbal and A. Ghafoor, "Analysis of multiband joint detection framework for waveform-based sensing in cognitive radios," in Proceedings of the 76th IEEE Vehicular Technology Conference (VTC Fall '12), pp. 1-5, September 2012.

[10] G. Huang and J. K. Tugnait, "On cyclostationarity based spectrum sensing under uncertain Gaussian noise," IEEE Transactions on Signal Processing, vol. 61, no. 8, pp. 2042-2054, 2013.

[11] W. Guibene, A. Hayar, M. Turki, and D. Slock, "A complete framework for spectrum sensing based on spectrum change points detection for wideband signals," in Proceedings of the IEEE 75th Vehicular Technology Conference (VTC '12), pp. 1-5, IEEE, Yokohama, Japan, May 2012.

[12] N. Zhang, H. Liang, N. Cheng, Y. Tang, J. W. Mark, and X. S. Shen, "Dynamic spectrum access in multi-channel cognitive radio networks," IEEE Journal on Selected Areas in Communications, vol. 32, no. 11, pp. 2053-2064, 2014.

[13] A. A. Khan, M. H. Rehmani, and M. Reisslein, "Cognitive radio for smart grids: survey of architectures, spectrum sensing mechanisms, and networking protocols," IEEE Communications Surveys and Tutorials, vol. 18, no. 1, pp. 860-898, 2016.

[14] D. Middleton, "Non-Gaussian noise models in signal processing for telecommunications: new methods an results for class A and class B noise models," IEEE Transactions on Information Theory, vol. 45, no. 4, pp. 1129-1149, 1999.

[15] K. Hassan, R. Gautier, I. Dayoub, M. Berbineau, and E. Radoi, "Multiple-antenna-based blind spectrum sensing in the presence of impulsive noise," IEEE Transactions on Vehicular Technology, vol. 63, no. 5, pp. 2248-2257, 2014.

[16] Q. Huang, P.-J. Chung, and J. Thompson, "A nonparametric approach for spectrum sensing using bootstrap techniques," in Proceedings of the IEEE Global Communications Conference (GLOBECOM '14), pp. 851-856, December 2014.

[17] A. Margoosian, J. Abouei, and K. N. Plataniotis, "An accurate kernelized energy detection in GAUssian and nonGAUssian/impulsive noises," IEEE Transactions on Signal Processing, vol. 63, no. 21, pp. 5621-5636, 2015.

[18] M. Shao and C. L. Nikias, "Signal processing with fractional lower order moments: stable processes and their applications," Proceedings of the IEEE, vol. 81, no. 7, pp. 986-1010, 1993. 
[19] R. Tandra and A. Sahai, "SNR walls for signal detection," IEEE Journal on Selected Topics in Signal Processing, vol. 2, no. 1, pp. 4-17, 2008.

[20] A. Mariani, A. Giorgetti, and M. Chiani, "Effects of noise power estimation on energy detection for cognitive radio applications," IEEE Transactions on Communications, vol. 59, no. 12, pp. 34103420, 2011.

[21] E. Xu and F. Labeau, "Impact evaluation of noise uncertainty in spectrum sensing under middleton class a noise," in Proceedings of the IEEE 12th Malaysia International Conference on Communications (MICC '15), pp. 36-40, Kuching, Malaysia, November 2015.

[22] S. M. Kay, Fundamentals of Statistical Signal Processing: Estimation Theory, Prentice-Hall Signal Processing Series, PrenticeHall, 1993.

[23] H. Urkowitz, "Energy detection of unknown deterministic signals," Proceedings of the IEEE, vol. 55, no. 4, pp. 523-531, 1967.

[24] J. Bond, S. Hui, D. Stein, and J. Zeidler, "A unified theory of adaptive locally optimum processing," in Proceedings of the Conference Record of the 27th Asilomar Conference on Signals, Systems and Computers, vol. 2, pp. 1594-1597, November 1993.

[25] A. Papoulis, Probability, Random Variables, and Stochastic Processes, McGraw-Hill, New York, NY, USA, 3rd edition, 1991.

[26] B. Picinbono, "On deflection as a performance criterion in detection," IEEE Transactions on Aerospace and Electronic Systems, vol. 31, no. 3, pp. 1072-1081, 1995.

[27] J. J. Lehtomäki, M. Juntti, H. Saarnisaari, and S. Koivu, “Threshold setting strategies for a quantized total power radiometer," IEEE Signal Processing Letters, vol. 12, no. 11, pp. 796-799, 2005. 

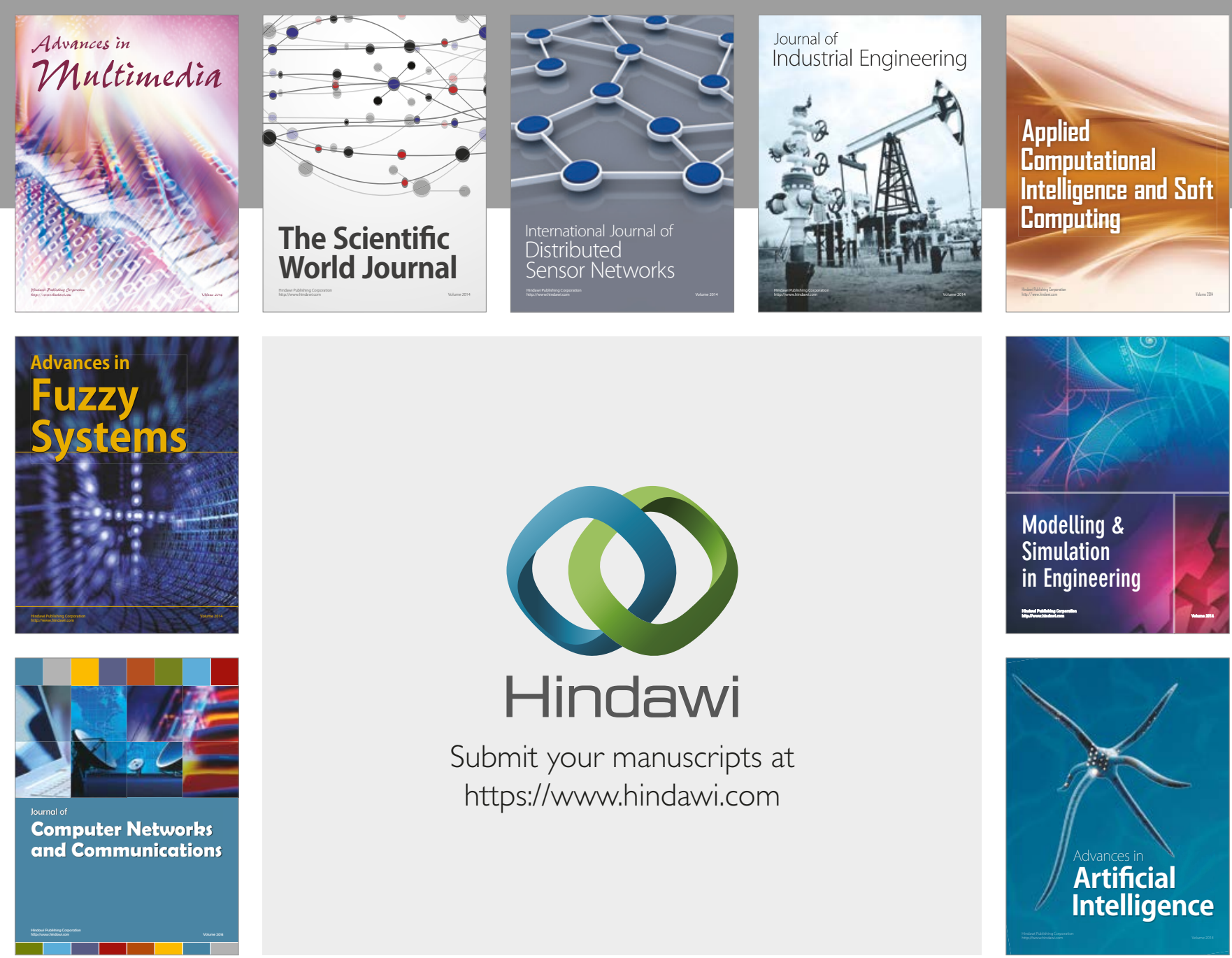

\section{Hindawi}

Submit your manuscripts at

https://www.hindawi.com
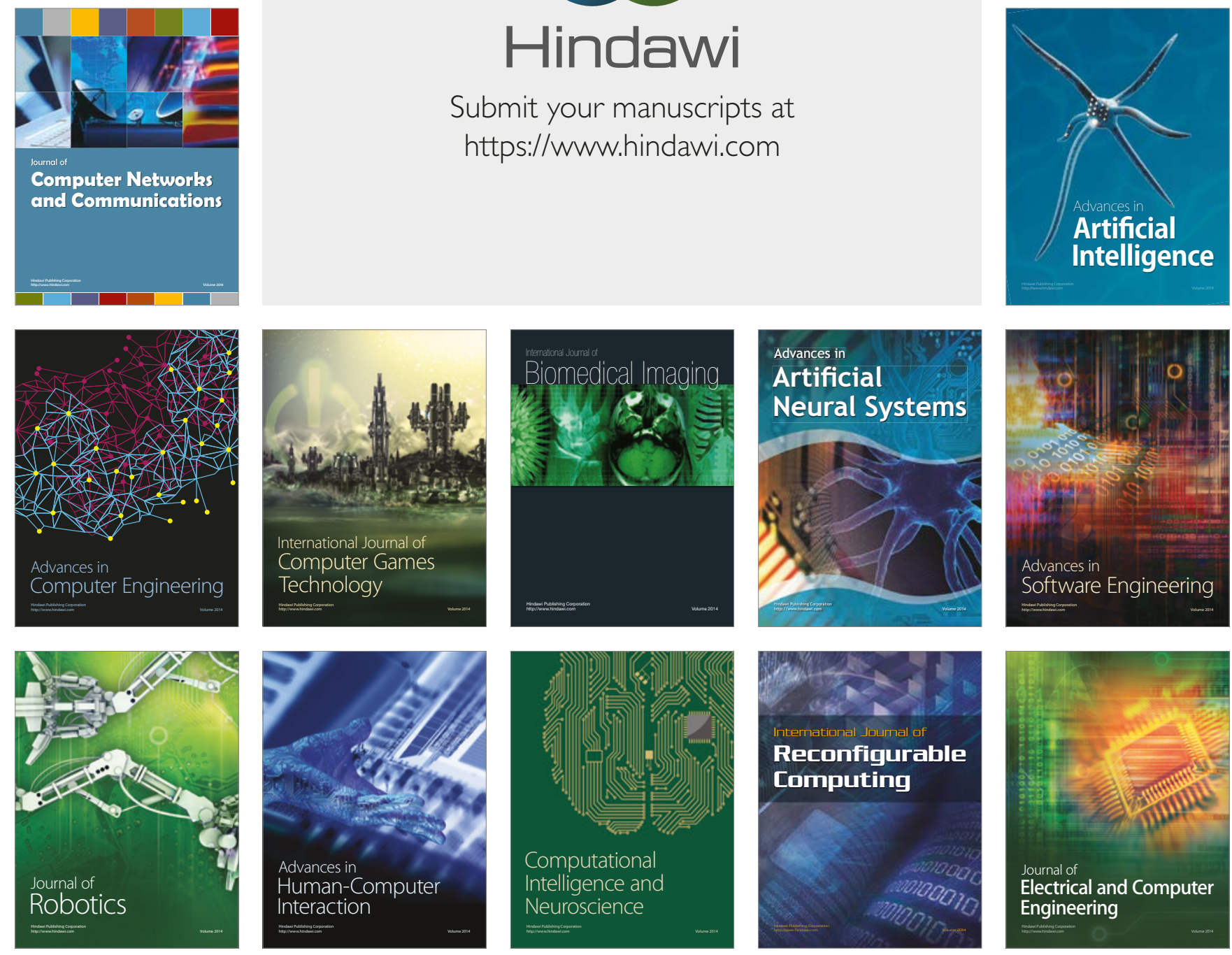